\title{
The benchtop mesoSPIM - a compact and versatile open-source light-sheet microscope for imaging large cleared tissue samples
}

Fabian F. Voigt ${ }^{1}$, Stéphane Pagès ${ }^{2}$, Martina Schaettin ${ }^{3}$, Anna Maria Reuss $^{4}$, Ruiyao $\mathrm{Cai}^{5}$, Shan Zhao ${ }^{6}$, Philipp Bethge ${ }^{1}$, Sven Hildebrand 7 , Anna Schueth ${ }^{7}$, Alard Roebrock ${ }^{7}$, Esther T Stöckli ${ }^{3}$, Laura Batti ${ }^{2}$, Ali Ertürk ${ }^{6}$, Adriano Aguzzi ${ }^{4}$, Fritjof Helmchen ${ }^{1}$ ${ }^{1}$ Brain Research Institute, University of Zurich, Switzerland. ${ }^{2}$ Wyss Center Geneva, Switzerland. ${ }^{3}$ Institute for Molecular Life Sciences, University of Zurich, Switzerland. 4 University Hospital Zurich, Switzerland. ${ }^{5}$ Stanford University, USA. ${ }^{6}$ Institute of Tissue Engineering and Regenerative Medicine, Helmholtz Center Munich, Germany. ${ }^{7}$ University of Maastricht, Netherlands

\section{Abstract Text}

Recently, we launched the mesoSPIM initiative (www.mesospim.org), an open-source project aimed at making light-sheet microscopes for imaging large (cm-sized) cleared tissue samples more accessible (Voigt et al., Nature Methods 2019). Here, we introduce the benchtop mesoSPIM, a more compact and cost-efficient version of the instrument which requires a budget of less than $100 \mathrm{k} €$ to build. Similar to the original mesoSPIM, the benchtop version utilizes an axially swept light-sheet to achieve near-isotropic resolution across a field of view up to $30 \mathrm{~mm}$. With a travel range of $50 \times 50 \times 100$ $\mathrm{mm}^{3}$ and a rotation stage, the instrument is capable of multi-view imaging of entire cleared mice. It is compatible with all clearing techniques and can image an entire mouse brain within 10 minutes at sub-cellular resolution. The microscope utilizes the same data acquisition software as the published instrument (github.com/mesoSPIM). We provide several application examples including screening APP/PS-1 mouse brains processed using the 
iDISCO protocol and imaging human cortex samples processed with an iDISCO/ECI/MASH protocol. The benchtop mesoSPIM is an attractive option for research groups and imaging facilities seeking a compact high-performance light-sheet microscope for cleared samples that can be adapted to a wide range of applications. 